\title{
Prognosis of Pregnancy and Childbirth in Heart Operated Patients: Experience in a 3 Referral Hospital in Mali, Case of the Gabriel University Hospital in Bamako
}

\author{
Fané Seydou1 ${ }^{*}$, Sanogo Siaka Amara1, Bocoum Amadou1, Sylla Checkna1, Kante Ibrahima², \\ Traore Youssouf ${ }^{1}$, Tegueté Ibrahima ${ }^{1}$, M. Traoré2 ${ }^{2}$ Mounkoro Niani ${ }^{1}$, A. Dolo ${ }^{1}$
}

${ }^{1}$ UTH Gabriel Toure, Bamako, Mali

${ }^{2}$ Point G Hospital Teaching, Bamako, Mali

Email: *Seydoufane@yahoo.fr

How to cite this paper: Seydou, F., Amara, S.S., Amadou, B., Checkna, S., Ibrahima, K., Youssouf, T., Ibrahima, T., Traoré, M., Niani, M. and Dolo, A. (2021) Prognosis of Pregnancy and Childbirth in Heart Operated Patients: Experience in a 3 Referral Hospital in Mali, Case of the Gabriel University Hospital in Bamako. Open Journal of Obstetrics and Gynecology, 11, 1875-1887. https://doi.org/10.4236/ojog.2021.1112174

Received: September 3, 2021

Accepted: December 28, 2021

Published: December 31, 2021

Copyright $\odot 2021$ by author(s) and Scientific Research Publishing Inc. This work is licensed under the Creative Commons Attribution International License (CC BY 4.0).

http://creativecommons.org/licenses/by/4.0/ (c) (i) Open Access

\begin{abstract}
Introduction: In sub-Saharan Africa and Mali, young women who have had heart surgery want to become pregnant. The occurrence of pregnancy in these women who have had heart surgery is becoming more and more frequent in our country because of the persistence of acute rheumatoid arthritis (RAA) and especially the increasingly easy access to heart surgery. General Objective: To study the evolution of pregnancy and the prognosis of childbirth in women who have undergone heart surgery. Methodology: This was a retrospective and descriptive study that took place over a period of five (5) years in the gynecology-obstetrics department of University Teaching Hospital (UTH) Gabriel Touré and the cardiology department of UTH Luxembourg. Was included in the study any pregnant woman admitted to the gynecology-obstetrics department of UTH Gabriel Touré and having a history of heart surgery. The variables studied were the socio-demographic characteristics, the type of heart disease, the management, the evolution of the pregnancy and the prognosis. Data was typed on word processor, Excel and analyzed on Epi info and SPSS. The Chi square or Fisher exact test (for the number $<$ to 5) and the relative risk (RR) with confidence interval (CI) to $95 \%$ were calculated. P was considered as significant if $<0.05$. Results: Of the 13,388 pregnant women admitted to the gynecology/obstetrics department of UTH Gabriel Touré, 20 pregnant women had a history of heart surgery $(1.49 \%$ ). The average age was 26 years old. The main cardiac pathology was valvular heart disease supported in $80.00 \%$ by the placement of a prosthesis.
\end{abstract}


During pregnancy follow-up, 55\% of pregnant women were on Anti-Vitamin $\mathrm{K}$ (AVK). In $95.00 \%$ of cases, heart disease was asymptomatic. We reported a case $(5.00 \%)$ of iterative cardiac decompensation, in which cardiac ultrasound found a very arrhythmic heart, grade III mitral leak, and massive aortic leak. We did not find any case of prosthetic thrombosis. The abortion rate was $5.00 \%$. The caesarean section rate was $31.60 \%$ and the instrumental extraction rate (forceps) was $23.10 \%$. Newborns had a normal birth weight (68.40\%), and were hypotrophic $(15.80 \%)$ and premature $(15.80 \%)$. In pregnant women on AVK, we reported 2 cases of fetal deaths in utero (10.00\%). Conclusion: Surgical treatment of operable heart disease is a real prophylaxis for gravidocardiac accidents. Pregnancy can be well tolerated in patients who underwent heart surgery with artificial heart valves.

\section{Keywords}

Cardiac Surgery, Pregnancy, Prognosis

\section{Introduction}

The association cardiopathy and pregnancy are common and some of these heart diseases require surgery (congenital malformations, severe rheumatoid arthritis). The management of heart disease during pregnancy poses two different problems, namely the evaluation and monitoring of pre-pregnancy cardiopathies which can be decompensated by the physiological changes induced by the latter and the peripartum [1]. The maternal morbidity associated with congestive heart failure, arrhythmia and vascular accidents is estimated between 8.00 and $30.00 \%$ and the fetal morbidity with increased prematurity and intrauterine growth retardation is estimated to $20.00 \%$ of pregnancies [1]. In Western industrialized countries, $0.20 \%$ to $4.00 \%$ of pregnancies are complicated by cardiovascular diseases, so in the United Kingdom, cardiovascular diseases are the leading cause of maternal deaths (2.31 per 100,000 pregnancies) and keep on increasing [2].

The management of these patients is based on a good knowledge of the physiological changes induced by pregnancy, as well as on a multidisciplinary approach as early as possible associating the cardiologist, the anesthesiologist, the resuscitator and the obstetrician gynecologist. In sub-Saharan Africa and Mali, rheumatoid arthritis is a common nosological group of cardiovascular pathologies [3]. Its treatment often requires the use of heart surgery often in women of childbearing age.

Indeed, the hemodynamic changes in pregnancy can unbalance in these pregnant women more or less precarious cardiac function [4]. In addition, the anticoagulant treatment to which patients with heart valves are subjected raises delicate therapeutic problems such as the risk of thromboembolism for the mother, the teratogenic risk for the fetus, and the risk of hemorrhage for the Moth- 
er-Fetus couple [4] [5]. Although the most complex situations are only documented by cases or series analyzed retrospectively or with observation, we have initiated this study to evaluate the evolution of the pregnancy in the heart operated pregnant women and establish the maternal-fetal prognosis.

\section{Materials and Method}

This was a retrospective and descriptive study that took place over a period of five (5) years from January $1^{\text {st }}, 2005$ to December $31^{\text {st }}, 2009$, in the gynecology-obstetrics department of Gabriel Touré University Teaching Hospital (UTH) and cardiology department of UTH Luxembourg. Was included in the study any pregnant woman admitted to the gynecology-obstetrics department of UTH Gabriel Touré and having a history of cardiac surgery. Not included in our study were all pregnant women who had no history of cardiac surgery or who had a history of cardiac surgery but who gave birth elsewhere or who refused to participate in the study or patient records were unusable.

The variables studied were the socio-demographic characteristics, the type of heart disease, the management, the evolution of the pregnancy; the maternalfetal prognosis.

The collection materials were: operative reports of cardiac surgery, prenatal check-books, birth records. The data was entered on Word, Excel and analyzed on epi info and SPSS. The Chi 2 test or Fisher's exact test (for numbers $<$ to 5) and the relative risk (RR) with its confidence interval to $95 \%$ were calculated; $p$ was considered significant if $<0.05$. Within the ethical framework, this present work has a purely scientific aim and intends to contribute to the fight against maternal-fetal morbidity and mortality which remain a real public health problem in developing countries, and more particularly in Black Africa. It guarantees the confidentiality of the data collected. The patients were informed of this confidentiality and freely consented to participate in the study.

\section{Results}

\subsection{Frequency}

We collected a total of 20 pregnant women who underwent heart surgery during the study period, with a prevalence of $0.14 \%(20 / 14285)$. There was an exponential evolution of the number of pregnant patients operated from the heart from 2 cases per year in 2005 to 7 cases in 2009.

\subsection{Socio-Demographic Data: (see Table 1)}

The average age of our pregnant women was 26 years with extremes of 17 years and 35 years. The 20 - 30 age group was the most represented with $85.00 \%$. All of our pregnant women were married, or $100 \%$. The primigravida represented $60.00 \%$, with 2 pregnancies of the extremes of one and 4 pregnancies. Nulliparous were in the majority $(60.00 \%)$ followed by primiparous $(20.00 \%)$ and pauciparous $(20.00 \%)$. 
Table 1. Socio-demographic characteristics.

\begin{tabular}{ccc}
\hline \multicolumn{1}{c}{ Parameter } & Number & Percentage \\
\hline Age & 17 & \\
$20-30$ years & 03 & $85.00 \%$ \\
$17-19$ years & & $15.00 \%$ \\
Gravidity & 12 & \\
Primigravida & 08 & $60.00 \%$ \\
Paucigravidy & & $40.00 \%$ \\
Parity & 12 & $60.00 \%$ \\
Nulliparous & 08 & $40.00 \%$ \\
Pauciparous & & \\
\hline
\end{tabular}

\subsection{Clinical Aspect: (See Table 2)}

Patients underwent cardiac surgery at the age group of 11 to 19 years in $70 \%$ and at age 30 and over in 5.00\%. Mitral heart valve diseases were the type of cardiopathy the most predominant (65.00\%) followed by Myxoma (10.00\%). The impairment was mono valvular $(87.50 \%)$ and poly valvular in $12.50 \%$.

The mitral prosthesis was the type of surgery performed $(80.00 \%)$ followed by the aortic prosthesis, patch CIV closure, atrial myxoma resection and CIA closure $(5.00 \%)$ each. The patients were on AVK in $65.00 \%$ before the beginning of pregnancy and in $55.00 \%$ during pregnancy. Other anticoagulants during pregnancy were heparin in $5.00 \%$ and $40.00 \%$ of the pregnant women were not on anticoagulant.

\subsection{Management and Prognosis during Pregnancy: (See Table 3)}

Throughout the pregnancy, 55.00\% of our pregnant patients were on AVK and that since the first trimester and $40.00 \%$ were not on anti-coagulant. Our pregnant women did antenatal visits to a midwife in $70.00 \%$ versus $30.00 \%$ who were seen by obstetricians. Regarding the evolution of pregnancy, $5 \%$ of pregnancies were complicated by spontaneous abortion and $5.00 \%$ by fetal malformations. We did not notice any maternal complications in $85.00 \%$ of our pregnant patients, however, we recorded one case of cardiac decompensation (5.00\%).

\subsection{Management and Prognosis of Delivery: (See Table 4)}

At the time of delivery, $42.1 \%$ of our pregnant patients were on AVK compared with $10.50 \%$ who were on heparin. Our patients delivered by caesarean section in $31.60 \%$ versus $68.40 \%$ by vaginal route including 3 cases of instrumental extractions. The indications for caesarean section were respectively Acute Fetal Distress (AFD) (33.30\%), BGR (16.60\%), heart failure (16.60\%) and operated heart $(33.30 \%)$. Immediate postpartum care was simple in $84.20 \%$ of our patients. Three patients $(15.80 \%)$ experienced impaired cardiac function, including an iterative cardiac decompensation with mitral insufficiency associated with 
Table 2. Clinical characteristics.

\begin{tabular}{lcc}
\hline \multicolumn{1}{c}{ Characteristics } & Number & Percentage \\
\hline Age at surgery & 14 & $70.00 \%$ \\
11 - 19 years & 06 & $30.00 \%$ \\
$\geq 30$ years & & \\
Type of cardiopathy & 10 & $50.00 \%$ \\
Mitral deficiency & 08 & $40.00 \%$ \\
Mitral closure & 02 & $10.00 \%$ \\
Myxoma & 14 & \\
Type of heart valve disease & 06 & $70.00 \%$ \\
Monovalvular & & $30.00 \%$ \\
Polyvalvular & 16 & \\
Type of surgery & 04 & $80.00 \%$ \\
Mitral prosthesis & & $20.00 \%$ \\
Aortic prosthesis & 13 & $65.00 \%$ \\
AVK before pregnancy & 07 & $35.00 \%$ \\
Yes & & \\
No & & \\
\hline
\end{tabular}

Table 3. Management and prognostic during pregnancy.

\begin{tabular}{lcc}
\multicolumn{1}{c}{ Elements } & Number & Percentage \\
Anticoagulant treatment & 11 & $55.00 \%$ \\
AVK & 02 & $10.00 \%$ \\
Heparin & 7 & $35.00 \%$ \\
Without anticoagulant & & \\
ANC provider & 06 & $30.00 \%$ \\
Obstetrician & 14 & $70.00 \%$ \\
Midwife & & \\
Pregnancy evolution & 04 & $20.00 \%$ \\
Spontaneous miscarriage & 16 & $80.00 \%$ \\
Normal & & \\
Maternal complications & 17 & $85.00 \%$ \\
None & 03 & $15.00 \%$ \\
Bad functioning tolerance & & \\
\hline
\end{tabular}

ANC: pregnancy monitoring

massive aortic insufficiency.

Regarding neonatal prognosis, we recorded $15.80 \%$ premature and $15.80 \%$ fetal hypotrophy. 
Table 4. Prognostic of delivery.

\begin{tabular}{|c|c|c|}
\hline Parameters & Number & Percentage \\
\hline \multicolumn{3}{|c|}{ Anticoagulant treatment during delivery } \\
\hline AVK & 08 & $42.10 \%$ \\
\hline Heparin & 02 & $10.50 \%$ \\
\hline Without anticoagulant & 9 & $45.00 \%$ \\
\hline \multicolumn{3}{|l|}{ Delivery mode } \\
\hline Caesarian & 06 & $30.00 \%$ \\
\hline Instrumental extraction & 04 & $20.00 \%$ \\
\hline Spontaneous & 10 & $50.00 \%$ \\
\hline \multicolumn{3}{|l|}{4 Indications of caesarian } \\
\hline AFD & 02 & $33.30 \%$ \\
\hline Cardiac insufficiency & 02 & $33.30 \%$ \\
\hline BGR & 01 & $16.60 \%$ \\
\hline Heart operated & 01 & $16.60 \%$ \\
\hline \multicolumn{3}{|l|}{ Postpartum care } \\
\hline Simples & 16 & $80.00 \%$ \\
\hline Impaired cardiac functioning & 04 & $20.00 \%$ \\
\hline \multicolumn{3}{|l|}{ Fetal prognostic } \\
\hline Stillbirths & 02 & $10.00 \%$ \\
\hline Hypotropia & 03 & $15.00 \%$ \\
\hline Preterms & 03 & $15.00 \%$ \\
\hline Fetal malformations & 01 & $05.00 \%$ \\
\hline Normal birth & 11 & $55.00 \%$ \\
\hline
\end{tabular}

AFD: Fetal asphyxia dystocia; BGR: Basin generally narrowed; AVK: Anti-vitamin K.

\section{Discussion}

\subsection{Methodological Approach}

This was a retrospective and descriptive study that took place over a period of five (5) years from January $1^{\text {st }}, 2005$ to December $31^{\text {st }}, 2009$ in two hospitals of Bamako district.

The difficulties during our study were essentially:

- the absence of the operative report of the cardiac surgery in the obstetrical file of the patients,

- the absence of direct contact of most patients,

- the absence of the cardiological management protocol in the obstetrical file.

\subsection{Frequency}

We collected a total of 20 pregnant women who underwent heart surgery during the study period with a prevalence of $0.14 \%$. M'baye and col. [5] had found a 
prevalence of $0.12 \%$ at DAKAR UTH in a series of 14 cases. ABDELLAOUI Y [6] in Fes and Assia AI [7] in Marrakech in Morocco reported a frequency of $1.20 \%$ and $3.20 \%$ respectively. Benzerdjeb B. [8] in Algeria reported a frequency of $0.19 \%$ in her series. The low prevalence in our series is explained by the difficulty of access to cardiac surgery in our country. In developed countries, heart valve diseases are becoming rarer in young women and the etiologies currently observed are predominantly degenerative and involve an older population [4]. In the literature, the association heart and pregnancy is about $1.00 \%$ [4] [9].

\subsection{Socio-Demographic Characteristics}

The age of the patient is largely involved in the assessment of maternal risk. The average age of our patients was 26 years, with extremes of 17 years and 35 years. This average is parallel to the data of the literature. Hanania et al. [10] in France and Iturbe-Alessio et al. [11] in Egypt reported an average age of 26 years and 27.7 years, respectively.

Assia Ai [7] and Abdellaoui Y [6] in Morocco reported respectively an average age of 30.24 years with extremes of 18 years and 41 years and 31 years with extremes of 22 years and 40 years. Benzerdjeb B. [8] in Algeria reported an average age of 32 years with extremes of 18 years and 44 years.

Multiparity may be the cause of some important obstetric complications such as Placenta Praevia (PP), Placental Hematoma (PH). The average gravidity of our pregnant women was 2 gravida and the average parity of 0.6 parous. M'baye and col. [5] had found a mean gravidity of 2.3 gravida with extremes of one and 12 for gravidity and an average parity of 0.60 parous. Abdellaoui Y [6], Assia Ai [7] in Morocco and Benzerdjeb B. [8] in Algeria reported respectively 26.90\%; $40.74 \%$ and $27.00 \%$ of primiparous.

\subsection{Clinical Aspect}

Heart diseases requiring surgical intervention should, in principle, have been made before pregnancy. However, surgery may be necessary during pregnancy if complications occur. The best time for correction of heart disease remains before pregnancy to avoid any fetal or maternal complications apart from the risks associated with surgery. All our patients were operated on before pregnancy.

The mean age at the time of cardiac surgery was 18.50 years with extremes of 11 years and 32 years. M'baye and col. [5] found an average age of 21.60 years in patients in their series at the time of valve replacement.

In our study, cardiac surgery was performed in $80.00 \%$ of the cases by a heart valve disease, in $10 \%$ of the cases by a myxoma of the atrium, in $5.00 \%$ of the cases respectively by an inter ventricular communication and an inter auricular communication. Mitral disease is generally well tolerated with possible occurrence of congestive heart failure. Mitral heart valve diseases were the most dominant in our study. They were isolated in $65.00 \%$ of cases, and associated in $15.00 \%$ of cases with an attack of other valves. Despite its high frequency in all 
series, the polyvalvular endocarditis represented $12.50 \%$ in our series to $42.32 \%$ in the study of Abdellaoui Y [6]. The mitral prosthesis was the type of surgery performed $(80.00 \%)$ followed by the aortic prosthesis, patch CIV closure, atrial myxoma resection and CIA closure (5.00\%) each. The progression of medical management by the broad application of antibiotic prophylaxis to acute rheumatic fever (ARF) and the progress of screening and early surgical treatment of congenital heart disease has made it possible to change the pattern of women with heart disease observed during pregnancy in Western countries with regression of heart valve disease of $20.00 \%$ in the UK or even $10.80 \%$ in Germany compared to congenital heart disease [12]. Abdellaoui Y [6] in Fez, in his series, reports a $30.7 \%$ history of cardiac surgery before pregnancy dominated by mitral and aortic prosthesis and the types of heart disease were heart valve diseases (92.32\%), including RM (53.86\%), IM (34.62\%) and congenital heart disease (3.84\%). Benzerdjeb B. [8] reported in her series 13 pregnant women operated from the heart over 22 cases of cardiopathies and pregnancy, or 59\%. The cardiac pathologies were dominated by heart valve diseases $61.0 \%$ (IM, RM, RA) congenital heart disease in $17.0 \%$ (CIA, CIV). These were essentially prosthetic surgeries [8]. Assia Ai [7] in Marrakech reported in her study 22.22\% history of cardiac surgery (18/81 patients) dominated by the mitral prosthesis (11.11\%) and the mechanical valve (9.87\%). M'baye and col. [5] reported that the mitral prosthesis was the dominant surgery (85\%).

\subsection{Management and Prognosis during Pregnancy}

A normal pregnancy imposes a regular obstetric follow-up schedule. However, a pregnant cardiac woman requires more rigorous follow-up and multidisciplinary care, including experienced cardiologists who must team up with obstetricians, anesthesiologists and neonatologists, with the aim of informing the patient of the maternal and fetal risks of pregnancy and establishing a clinical and cardiographic examination with an adaptation of the medical treatment and rarely asking the surgical indication. Patients had antenatal visits to a midwife in $70.00 \%$ versus $30.00 \%$ who were seen by obstetricians. A poor frequency compared with the risk incurred in parturient women with heart disease. This may be due to either difficulties of access to care, economic problems or failure to raise the patient's awareness of the risks. Assia Ai [7] and Abdellaoui Y [6] reported in their studies that patients were not followed in respectively $30.86 \%$ and $42.30 \%$. The treatment of a cardiopathy in a pregnant woman is a challenge sometimes difficult. An evaluation is necessary for the expected benefit and the potential risk for the child. No therapeutic substance prescribed during pregnancy can be considered totally harmless. During the whole pregnancy, $55.00 \%$ of our pregnant women were on AVK and that since the $1^{\text {st }}$ trimester and $40.00 \%$ were not under anti-coagulant. In contrast, $45.67 \%$ of the pregnant women were on anticoagulant and $12.34 \%$ on digitalis in the study of Assia Ai [7]. In the study of Abdella Y [6] 23.07\% were on anticoagulant. Pregnancy is responsible for a state 
of hypercoagulability that increases the risk of thrombosis. So, the problem is not so much the haemorrhagic risk as the thromboembolic risk. The risk of maternal and fetal hemorrhage exists throughout pregnancy, but also during delivery and postpartum. At the obstetric level, standard care had been taken, with an average of one antenatal consultation per month. Most patients were received during the $2^{\text {nd }}$ trimester of pregnancy and did not benefit from a relay of AVK treatment by heparinotherapy during the first quarter period. Only $5.00 \%$ of our pregnant women had benefited from a relay of AVK treatment by subcutaneous route during the first trimester period. $10.50 \%$ of our pregnant women had benefited from AVK treatment with heparin, reintroduced during the last two weeks of pregnancy. This heparinotherapy was discontinued at the beginning of labor and resumed six to ten hours after delivery. Low molecular weight heparins were the most used. Thus, Enoxaparin (Lovenox) was used at a dosage of 4000 IU every 12 hours. This protocol contrasts with the literature [13] which advises a relay of AVK by heparin two weeks before the expected date of delivery. Our results could be explained by the fact that $60 \%$ of our pregnant women were attended by midwives in antenatal care. In our study, only one pregnant woman, $5 \%$ of the series had metrorrhagia, without hemodynamic consequences in the $1^{\text {st }}$ trimester. The pregnant woman was on heparin. Iturbe-Alessio et al. [11] found $13.20 \%$ hemorrhage under heparin, while M'baye et al. [5] found $42.80 \%$ in their series.

Spontaneous abortions are more common with anticoagulants. In our series we pointed out $5.00 \%$ of cases of spontaneous abortions. Thomas D. et al. [14] and Assia $\mathrm{Ai}$ [7] reported respectively $19.00 \%$ and $3.70 \%$ spontaneous abortions in their series, compared to $10.00 \%$ in the general population. Our result is lower than this result. This may be explained by the small size of our sample but also by the fact that all our patients were not under anticoagulant.

Maternal risks depend on the nature of the cardiopathy and its pre-conception functional tolerance, which may range from deteriorating ventricular function to heart failure or even death. Heart disease was most often asymptomatic during pregnancy; however, we noted three cases of dyspnea (15.00\%) including one case $(5.00 \%)$ of cardiac decompensation iterative in whom cardiac ultrasound had found a very arrhythmic heart, a grade III mitral leak, and a massive aortic leak requiring a proposal for double valvular substitution. M'baye and col. [5] found $21.40 \%$ heart failure. In their series, Hanania et al. [10], Salazar et al. [15] figured out respectively $2.20 \%$ and $2.00 \%$ heart failure. Abdellaoui Y [6] in his series reported 13 cases of complication (50.00\%) of which 12 developed heart failure and 1 case of PAH. Assia Ai [7] pointed out in her study that 32 women, or $39.50 \%$ of parturients, had complications during pregnancy dominated by heart failure and cardiogenic shock.

In our study, we did not find any case of prosthetic thrombosis. In the literature, the percentage of prosthetic thrombosis varies between $0.00 \%$ and $9.20 \%$ [15] [16]. Our results could be explained by the use of little thrombogenic prosthesis, Saint-Jude type. Thromboembolic events, however, were high in other 
studies such as that of Hanania et al. [10] where they were multiplied by ten. The risk of thromboembolism was 4.5 times higher with heparin than with AVK, as found in the Iturbe-Alessio et al. [11] where the three cases of prosthetic thrombosis all occurred with heparin.

\subsection{Management and Prognosis of Childbirth}

The question that always arises regarding cardiac parturients is the mode of delivery of the patients and the type of adequate anesthesia. Vaginal delivery remains the preferred delivery mode for most women with heart disease unless there are specific obstetric indications.

In our study, $31.60 \%$ of pregnant women gave birth by caesarean section and $68.4 \%$ delivered vaginally, of which $23.1 \%$ benefited from forceps assisted delivery. This result is lower than that reported by M'baye et al. [5] which was 77.70\%. Assia Ai [7], Benzerdjeb B. [8] and Abdellaoui Y [6] reported respectively $51.8 \%$; $35 \%$ and $72.73 \%$ vaginal delivery. None of our parturients has benefited from epidural anesthesia, however Abdellaoui Y [6] reports 12.5\% in her series. Our caesarean section rate is similar to data from the literature [6] [7] [8] who reported $48.15 \%, 65.00 \%$ and $27.27 \%$ respectively.

The indications in $50.00 \%$ of women having caesarian section are strictly cardiac and compatible with the literature [6], while $50 \%$ of women who underwent caesarian section are for obstetric causes. We observed no complication at the time of delivery, the same observation was made by Benzerdjeb B. [8]

However, in the postpartum, the main complications for these women are mainly hemorrhagic and thromboembolic in parturients under anticoagulant and those with mechanical valves. Hemodynamic complications remain rare and can be prevented in some situations by surgical or interventional correction during pregnancy or per-partum. The risk of infection is low given the protocols of antibiotic prophylaxis.

In our study, one patient developed an iterative cardiac decompensation, in which cardiac ultrasound revealed a very arrhythmic heart, grade III Doppler mitral leak, massive aortic leak, and dilated left ventricle. This required a proposal for a double mitro-aortic valve replacement. Abdellaoui Y [6] did not find any postpartum complications in her series, however Assia Ai [7] reported that $30.8 \%$ of patients had post-partum complications dominated by cardiac decompensation (19.75\%) and thromboembolic events (6.17\%).

We did not notice any haemorrhage of the delivery, nor haemorrhage in the postpartum. Lee PK et al. [17] reported two cases of massive bleeding of the delivery. This result contrasts with ours and may be accounted for by the size of our sample which is lower than that of Lee PK et al. [17]. We did not also have maternal deaths in our series, however maternal mortality was $12.3 \%$ in the series of Assia $\mathrm{Ai} \mathrm{[7]} \mathrm{with} \mathrm{heart} \mathrm{valve} \mathrm{diseases} \mathrm{(8.6 \% );} \mathrm{peripartum} \mathrm{cardiomyopathy}$ (2.46\%) and dilated cardiomyopathy (1.23\%). Our results are parallel to those of Vitale [18] on a series of 58 pregnancies. Iturbe-Alessio et al. [11], Born et al. [19], Larrea et al. [20] found respectively $2.7 \%, 5.7 \%$, and $2.1 \%$ of deaths. M'baye 
and col. [5] found $7.1 \%$ of deaths in their series.

The fetal risks are related either to the existence of a maternal cyanosis leading to an insufficiency of oxygenation responsible for abortion, prematurity, IUGR, or a deterioration of the maternal cardiac function responsible for a circulatory insufficiency of the placenta. In our study, we recorded 19 newborns, including 3 cases of premature babies (15.80\%); 3 cases of hypotrophy (15.80\%). In our series, we noted two cases of fetal death in utero (10.00\%) and their mothers were all under AVK throughout the pregnancy. Our prematurity rate is similar to that of Abdellaoui Y [6] (12.00\%) and our fetal mortality rate is lower than that of Assia Ai [7] (23.5\%). This difference can be explained by the size of our sample of 22 patients versus 81 patients for Assia Ai.

The only case of fetal malformation we had, was in a mother who was not on anticoagulation, but who was the oldest in the series. Coumarin embryopathies vary widely according to the literature, ranging from $0 \%$ to $44 \%$ [15]. Sareli et al. [21] and Iturbe-Alessio et al. [11] reported in their series $4 \%$ and $28.5 \%$ respectively. However, coumarin embryopathies have been reported in series where high doses of AVK have been used [5]. Most of our patients were under minisintrom dosed at $1 \mathrm{mg}$. It has been shown that when the dosage of warfarin is less than or equal to $5 \mathrm{mg} /$ day, the incidence of coumarin embryopathy decreases significantly or even nil [18]. Our results are in agreement with those of Hanania et al. [10].

\section{Conclusions}

At the end of our five-year retrospective study, we found out that in Mali, cardiac surgery is mostly performed during adolescence and that cardiac surgery is largely dominated by heart valve diseases.

Despite the lack of pre-conception management, pregnancy can be well tolerated in patients with heart operated surgery and artificial valves.

The management of these parturients must be multidisciplinary, in collaboration between cardiologist, obstetrician, resuscitator and neonatologist; based on a good understanding of the risks specific to each pathology.

The planning of pregnancy and a maximum avoidance of acute decompensations, would improve the maternal-fetal prognosis.

The surgical treatment of operable cardiopathies is a real prophylaxis for gravidocardiac accidents.

\section{Conflicts of Interest}

The authors declare no conflicts of interest regarding the publication of this paper.

\section{References}

[1] Siu, S.C., Sermer, M., Colman, J.M., Alvarez, A.N., Mercier, L.-A., Morton, B.C., et al. (2001) Prospective Multicenter Study of Pregnancy Outcomes in Women with Heart Disease. Circulation, 104, 515-521. https://doi.org/10.1161/hc3001.093437 
[2] Jastrow, N., Meyer, P., Bouchardy, J., Savoldelli, G.L. and Irion, O. (2011) Maternal Heart Disease and Pregnancy Multidisciplinary Management. Revue Médicale Suisse, 7, 2070-2077.

[3] Ba-Ba, K.A., Hane, L., Diop, I.B., Sarr, M., Bao, O., et al. (1998) Rheumatic Heart Disease in Senegal: Clinical and Therapeutic Aspects. Tropical Cardiology Journal, 24, 316-336.

[4] Luthra, A., Bajaj, R., Jafra, A., Jangra, K. and Arya, V.K. (2017) Anesthesia in Pregnancy with Heart Disease. Saudi Journal of Anaesthesia, 11, 454-471. https://doi.org/10.4103/sja.SJA $277 \quad 17$

[5] Mbaye, M., Moreira, P.M., Guèye, S.M., Cissé, C.T., Moreau, J.C., Kane, A. and Diao, M. (2009) Pregnancies Associated with Valvular Prosthesis at Dakar Teaching Hospital: Prognosis, Epidemiological, Clinical and Therapeutical Aspects. Journal de Gynécologie Obstétrique et Biologie de la Reproduction, 38, 83-88. https://doi.org/10.1016/j.jgyn.2008.09.007

[6] Abdellaoui, Y. (2012) Cardiopathies and Pregnancy about 26 Cases. Thesis of Medicine No. 115, Sidi Mohammed Ben Abdellah University, Fez, 138 p.

[7] Assia, A. (2016) The Management of Cardiac Parturients in Intensive Care about 81 Cases. Medical Thesis No. 91, Caddi Ayyad University, Marrakech, 136 p.

[8] Benzerdjeb, B. (2015) Pregnancy in Women with Heart Disease. End of Study Dissertation in Medicine. Abou Bekr Belkaid University, Tlemcen, 136 p.

[9] Samba Hann, B. (2005) Pregnancy in Women with Heart Disease. Retrospective Study of 50 Cases Collected at the Cardiology Clinic of the University Teaching Hospital Aristide-Le-Dantec. Memory Certificate of Special Studies in Cardiology. University of Dakar, Dakar, $150 \mathrm{p}$.

[10] Hanania, G. and Nassivera, L. (2001) Conduct Anticoagulant Treatment during Pregnancy in Patients with Mechanical Prosthesis. Heart Disease Archives and Vessels, 10, 1073-1076.

[11] Iturbe-Alessio, I., Del Carmen, C., Mutchinik, O., Santos, M.A., Zajarías, A. and Salazar, E. (1986) Risk of Anticoagulant Therapy in Pregnant Women with Artificial Heart Valves. The New England Journal of Medicine, 315, 1390-1393. https://doi.org/10.1056/NEJM198611273152205

[12] Am, M. (1995) Pregnancy in the Women with Congenital Heart Disease. American Journal of Cardiac Imaging, 1, 44-52.

[13] Bernard, J., Duffour, P., Subtil, D., Vaksmann, S., Monier, S., Puech, F., et al. (1997) Pregnancies in Mechanical Cardiac Valve Carriers. Literature Paper. Journal de Gynécologie Obstétrique et Biologie de la Reproduction, 26, 455-464.

[14] Thomas, D., Boubrit, K., Darbois, Y., Seebacher, J., Seirafi, D. and Hanania, G. (1994) Pregnancies in Cardiac Valve Carriers. Retrospective Study about 40 Pregnancies. Annales de Cardiologie et d' Angéiologie, 43, 313-321.

[15] Salazar, E., Zajarias, A., Guttiaraz, N. and Iturbe, I. (1984) The Problem of Cardiac valve Prostheses, Anticoagulant, and Pregnancy. Circulation, 70, 169-177

[16] Born, D., Martinez, E.E., Almeida, P.A.M., Santos, D.V., Carvalho, A.C.C., Moron, A.F., et al. (1992) Pregnancy in Patients with Prosthetic Heart Valves: The Effect of the Anticoagulant on Mother Fetus, and Neonate. American Heart Journal, 124, 413 417. https://doi.org/10.1016/0002-8703(92)90606-V

[17] Lee, P.K., Wang, R.Y.C., Chow, J.S.F., Cheung, K.-L., Wong, V.C.W. and Chan, T.-K. (1986) Combined of Warfarin and Subcutaneous Heparin in Patients with an Artificial Heart Valve. Journal of the American College of Cardiology, 8, 221-224. https://doi.org/10.1016/S0735-1097(86)80116-4 
[18] Adebola, P.A., Daniel, F.A., Oshodi, Y.A., Gbadegesin, A., Ale, O.K., Oluwole, A.O., Falase, O., Oludara, M.A. and Nwiloh, J. (2021) Anticoagulation in Pregnant Women with Mechanical Heart Valve Prostheses: Case Reports and a Literature Review. Nigerian Postgraduate Medical Journal, 28, 68-70.

https://doi.org/10.4103/npmj.npmj $344 \quad 20$

[19] Salazar, E., Zajarias, A., Guttiaraz, N. and Iturbe, I. (1984) The Problem of Cardiac Valve Prostheses, Anticoagulant, and Pregnancy. Circulation, 70, 169-177.

[20] Larrea, J.L., Nunez, L., Reque, J.A., Gil Aguado, M., Matarros, R. and Minguez, J.A. (1983) Pregnancy and Mechanical Valve Prostheses: A High Risk Situation for the Mother and the Fetus. The Annals of Thoracic Surgery, 36, 459-463.

https://doi.org/10.1016/S0003-4975(10)60488-2

[21] Sareli, P., England, M.J., Berk, M.R., Marcus, R.H., Epstein, M., Driscoll, J., et al. (1987) Maternal and Fetal Sequelae of Anticoagulation during Pregnancy in Patients with Mechanical Heart Valve Prostheses. The American Journal of Cardiology, 63, 1462-1465. https://doi.org/10.1016/0002-9149(89)90008-8 